\title{
Adolescência e organização de personalidade borderline: caracterização dos vínculos afetivos ${ }^{1}$
}

\author{
Aline Bedin Jordão ${ }^{2}$ \\ Universidade Federal de Santa Maria, Santa Maria-RS, Brasil \\ Vera Regina Röhnelt Ramires \\ Universidade do Vale do Rio dos Sinos, São Leopoldo-RS, Brasil
}

\begin{abstract}
Resumo: A demanda clínica atual inclui um número significativo de pacientes borderlines. Esse estudo buscou realizar uma revisão de literatura abrangente e não sistemática sobre os vínculos afetivos de adolescentes com indicadores de personalidade borderline. Foram consultadas bases de dados nacionais e internacionais, privilegiando-se publicações a partir de 1995, no referencial psicanalítico. Os estudos encontrados foram discutidos em três grupos: adolescência e cultura contemporânea, características da organização borderline na adolescência e vínculos afetivos desses adolescentes. Discute-se a importância dos vínculos de apego inseguro, a questão da psicopatologia materna e o papel das experiências traumáticas na história de vida desses pacientes.
\end{abstract}

Palavras-chave: adolescentes, comportamento de apego, distúrbio da personalidade borderline.

\section{Adolescence and borderline personality disorder: characterization of affective bonds}

\begin{abstract}
Currently, there are a large number of borderline patients in health services. This is a comprehensive and nonsystematic literature review on adolescents' emotional bonds and borderline personality disorder. National and international databases were searched, and publications addressing the psychoanalytic theory since 1995 were analyzed. The identified studies were discussed in three groups: adolescents and contemporary culture; features of borderline organization in adolescence; and adolescents' affective ties. The importance of insecure attachment, maternal psychopathology and the role of traumatic experiences in these patients' lives are discussed.
\end{abstract}

Keywords: adolescents, attachment behavior, borderline personality disorder.

\section{Adolescente y transtorno de personalidad limitrofe: caracterización de los vínculos afectivos}

\begin{abstract}
Resumen: Hoy la demanda clinica incluye muchos pacientes borderline. Este estudio realiza una revisión exhaustiva y no sistemática de la literatura sobre los vínculos emocionales de los adolescentes borderline. Se consultó a las bases de datos nacionales e internacionales, con preferencia a las publicaciones a partir de 1995, en las referencias psicoanalíticas. Los estudios se examinaron en tres grupos: los adolescentes y la cultura contemporánea, las características de la organización borderline en la adolescencia y vínculos emocionales de los adolescentes. Se discute la importancia del apego inseguro, la psicopatología materna y el papel de las experiencias traumáticas en la historia de la vida.
\end{abstract}

Palabras clave: adolescente, conducta de apego, trastorno de personalidad limitrofe.

Este artigo apresenta uma revisão de literatura abrangente e não sistemática sobre o tema dos vínculos afetivos estabelecidos entre adolescentes com indicadores de organização de personalidade borderline e seus pais (ou principais cuidadores). Vários autores, entre eles Green (1977) e Outeiral (1993), referem que a demanda clínica atual inclui um número significativo de pacientes borderlines, em contraste

\footnotetext{
${ }^{1}$ Este trabalho é derivado da Dissertação de Mestrado defendida pela primeira autora, sob a orientação da segunda, no Programa de Pósgraduação em Psicologia Clínica da Universidade do Vale Rio dos Sinos, São Leopoldo, RS. Este texto foi revisado segundo Acordo Ortográfico da Língua Portuguesa (1990), em vigor a partir de $1^{\circ}$ de janeiro de 2009.

${ }^{2}$ Endereço para correspondência:

Aline Bedin Jordão. Clínica de Estudos e Intervenções em Psicologia (CEIP) da Universidade Federal de Santa Maria (UFSM). Rua Floriano Peixoto, 1750. Prédio de Apoio da Universidade Federal de Santa Maria. Térreo, sala 01. CEP 97.015-372. Santa Maria-RS, Brasil. E-mail: alinejor@terra.com.br
}

com períodos anteriores em que as psiconeuroses eram predominantes. Além disso, o interesse pelo tema alicerça-se no pressuposto de que o desenvolvimento psicológico acontece em uma matriz intersubjetiva e os vínculos familiares ocupam, portanto, um lugar de destaque neste processo (Berenstein, 2003; Kaës, Faimberg, Enriquez, \& Barane, 1993/2001).

Kernberg (1995) identifica três grandes organizações estruturais correspondentes à personalidade neurótica, borderline e psicótica. Na organização borderline descreve uma falta constitucional de autonomia primária, baixa tolerância à ansiedade, importante desenvolvimento dos impulsos agressivos e vivências de uma realidade excessivamente frustrante. Para esse autor, três critérios estruturais, correspondentes à organização intrapsíquica, permitiriam o diagnóstico psicodinâmico da organização de personalidade borderline: a difusão da identidade, o predomínio de defesas baseadas na dissociação e a conservação da prova de realidade. 
Critérios adicionais descritos por Kernberg (1995) incluem manifestações não específicas de fragilidade egóica (falta de tolerância à ansiedade, controle de impulsos e capacidade de sublimação), graves perturbações nas relações de objeto, e sintomas neuróticos múltiplos e crônicos (fobias, obsessões e ansiedade). Incluem também uma falta de integração do superego com tendências anti-sociais, predomínio do processo primário e condensação de conflitos edípicos e pré-edípícos.

Em termos de diagnóstico descritivo, o Manual Diagnóstico e Estatístico de Transtornos Mentais (American Psychiatric Association, 1995) aponta nove critérios para o "Transtorno de Personalidade Borderline", sendo cinco suficientes para sua identificação. São eles: um padrão de relacionamentos interpessoais instáveis e intensos; esforços frenéticos para evitar um abandono real ou imaginado; perturbação da identidade; impulsividade; ameaças suicidas ou comportamento automutilante; instabilidade afetiva; sentimentos crônicos de vazio; raiva e dificuldade para controlar a raiva; e ideação paranóide transitória.

Em contrapartida, Skodol e cols. (2002), embora reconhecendo as vantagens dos critérios descritivos, salientam que outros importantes aspectos da psicopatologia desse transtorno devem ser incluídos. Entre esses, citam a co-morbidade com os transtornos do Eixo I e outras desordens do Eixo II, além da heterogeneidade dentro do diagnóstico e de outras possíveis características da estrutura da personalidade. Esses autores apontam ainda, da mesma forma que Gunderson (2008), as bases genéticas do transtorno como uma dimensão a ser considerada nos processos subjacentes à falta de regulação do afeto, ao comportamento impulsivo e aos distúrbios interpessoais, característicos do transtorno.

A importância dos distúrbios nos vínculos afetivos da organização de personalidade borderline foi salientada por diversos autores, como se verá mais adiante. Agrawal, Gunderson, Holmes e Lyons-Ruth (2004), em um artigo de revisão, demonstraram que muitos estudos concluíram que há uma forte associação entre apego inseguro e organização de personalidade borderline. Isto, para os autores, constituiria uma direção para futuras pesquisas.

Nessa linha, essa revisão de literatura foi realizada com o objetivo de analisar o tópico dos vínculos afetivos de adolescentes com indicadores de personalidade borderline. A literatura revisada insere-se no campo da teoria psicanalítica, em especial no que diz respeito à psicodinâmica dos adolescentes com organização borderline e suas famílias, bem como as vicissitudes dos seus vínculos afetivos, em particular os de apego. Buscou-se livros e artigos, sendo que as bases de dados consultadas foram PsycInfo, BVS, SciELO, PEPSIC e Academic Search Premier. Os descritores utilizados para a busca de artigos foram: "organização de personalidade borderline", "adolescente borderline", "adolescência e personalidade borderline", "vínculos familiares e adolescência" e "vínculos afetivos e organização borderline". Os livros considerados relevantes para análise dos vínculos afetivos e a organização de personalidade borderline foram incluídos, independentemente do ano de sua publicação. Quanto aos artigos, priorizaram-se publicações a partir do ano de 1995.

De acordo com o conteúdo abordado, os estudos encontrados foram organizados em três tópicos: adolescência e cultura contemporânea, a organização da personalidade borderline na adolescência e as características dos vínculos afetivos dos adolescentes com organização de personalidade borderline. Esses tópicos compõem as seguintes seções desse artigo.

$\mathrm{O}$ interesse pela adolescência justifica-se pelo fato de que, de acordo com a vertente psicanalítica, o adolescente vivencia situações complexas: desidentificações, neoidentificações, difusões identitárias, oscilações entre a busca da dependência e a necessidade de estabelecer sua individuação, confrontação de gerações, dentre outras. Revisitar os ideais narcísicos parentais, diferenciar-se e subjetivarse se afiguram como desafios significativos deste processo (Calligaris, 2000; Jeammet \& Corcos, 2005; Kancyper, 1999). A compreensão dos processos característicos desse período pode subsidiar tanto as intervenções no campo da clínica psicológica, como ações de prevenção e promoção da saúde mental dessa população.

Além disso, considera-se que o período da adolescência, por si só, já se configura como uma situação-limite, tendo em vista todos os enfrentamentos necessários e todas as reorganizações subjetivas daí decorrentes. Por caracterizarse como um período emocionalmente turbulento, marcado por ressignificações e movimentações pulsionais intensas, entende-se que das derivações dessas turbulências é que serão definidas as características mais fixas e estáveis da personalidade (Baird, Veague, \& Rabbitt, 2005).

Jeammet e Corcos (2005), nesse sentido, referem que quanto mais a dependência é evitada e intolerável, social e subjetivamente, mais ela terá que ser expressa em sua forma mais "crua", mediante os sintomas dos adolescentes de hoje. Afirmam, ainda, que o terreno psíquico (com suas representações simbólicas e afetivas) perde para as dimensões do campo sensorial e perceptivo nas manifestações da adolescência. Assim, as relações de dependência associamse com a predominância das "patologias do agir", tais como as toxicomanias, o alcoolismo, as condutas autodestrutivas, o uso abusivo de medicamentos, os transtornos alimentares (anorexia e bulimia) e as demais manifestações borderline.

A capacidade reflexiva e a possibilidade de simbolização aparecem como essenciais no processo adolescente. Dar sentido às vivências e nomear as turbulências e mudanças experimentadas permite uma reestruturação psíquica satisfatória (Fonagy \& Target, 2004; Graña, 2007; Leivi, 1995; Levisky, 2002; Savietto, 2007; Savietto \& Cardoso, 2006). Em se tratando dos adolescentes com organização borderline, toda esta dinâmica revela-se ainda mais intensificada. Adquire relevância, portanto, a dimensão da história de vida destes adolescentes, geralmente marcadas por funcionamentos 
familiares bastante comprometidos, denotando um contexto instável, caótico, inseguro. Assim, experiências traumáticas, negligências, abusos, violências das mais diversas ordens têm sido apontadas como precursores do desenvolvimento de uma organização de personalidade borderline na adolescência (Bradley \& Westen, 2005; Carvalho, 2004; Figueiredo, 2003; Reich \& Zanarini, 2001; Russ, Heim, \& Westen, 2003; Villa \& Cardoso, 2004) e justificam o interesse desse estudo.

\section{Adolescência e cultura contemporânea}

Para além das questões intrapsíquicas e intersubjetivas, fundamentais para a constituição de uma personalidade saudável, a literatura também discute a influência da cultura contemporânea neste processo e suas interferências nas vivências da adolescência. O tempo contemporâneo é marcado pelo instantâneo, pela superficialidade e pelo imediatismo que (des)estrutura as relações (Cardoso, 2005; Figueiredo, 2003; Justo, 2005).

Soma-se a isso a questão de que, culturalmente, ser adolescente é um ideal e a mídia contribui significativamente para a representação deste ideal: ser jovem, transgredir, não ter limites, estar "fora da lei" (Calligaris, 2000; Savietto, 2007). Assim, as barreiras intergeracionais cada vez diminuem mais, o que deixa o adolescente em uma situação de desamparo, sem uma figura de adulto para se identificar (Jeammet \& Corcos, 2005). Kehl (2003), discutindo o fenômeno de "teenagização da cultura ocidental" e das mudanças nas configurações familiares, refere que o abandono das crianças e adolescentes de hoje ocorre quando os adultos responsáveis não sustentam sua diferença diante deles, não lhes conferindo um lugar. Tal situação resulta na fragilidade da transmissão de parâmetros éticos para as novas gerações.

Com efeito, o "fenômeno borderline" articula-se ao contexto da atualidade, tendo em vista este mal-estar contemporâneo, marcado por uma cultura cada vez mais competitiva, individualista e narcísica. Como conseqüência, aparece o "desenraizamento" do ser humano e o aumento de seu desamparo. O adolescente borderline, assim, busca um lugar de acolhimento cada vez mais difícil de encontrar (Cardoso, 2005; Figueiredo, 2003; Hegenberg, 2000). Todos estes fatores, somados à proliferação da violência nos mais diversos âmbitos (urbano, familiar, institucional), à crise de valores éticos e às novas configurações familiares não podem ser desprezados quanto às suas implicações em termos de sofrimento psíquico e repercussões nos vínculos afetivos durante a adolescência (Birman, 2005; Levisky, 2002; Roudinesco, 2003; Savietto, 2007; Savietto \& Cardoso, 2006).

Diante desse panorama, os pais encontram-se "desmapeados" e, conseqüentemente, os filhos "desamparados" (Savietto, 2007; Savietto \& Cardoso, 2006). Como repercussão, aparece o acréscimo das manifestações psicopatológicas entre os adolescentes, expressas por meio do registro do ato (atuações) e da convocação do corpo, o que supõe uma precariedade dos mecanismos de simbolização (Figueiredo, 2003; Fonagy \& Target, 2004; Levisky, 2002; Savietto \& Cardoso, 2006).

Nessa vertente, Roudinesco (2003) salientou a 'desordem' em algumas dinâmicas familiares no cenário atual, em que a hierarquia parece não ter mais sentido, já que o poder se encontra descentralizado, caracterizando uma família "fraterna", igualitária. Como conseqüência, aparece o enfraquecimento das referências parentais e a diluição dos limites e das regras, o que, muitas vezes, se traduz em desamparo, sobre-excitação e tendência a descargas afetivas na adolescência (Savietto, 2007).

Pode-se associar estas situações com as manifestações borderline, tendo em vista que nos adolescentes com este funcionamento a vivência afetiva apresenta-se desorganizada, com vinculações afetivas comprometidas, senso de identidade fragilizado e dinâmicas familiares marcadas por apegos do tipo inseguro. Além disso, as atuações (acting out) aparecem em relevo, podendo-se associar à insuficiência na rede de apoio e na sustentação afetiva percebida por estes adolescentes para o desenvolvimento de seu self (Bradley \& Westen, 2005; Fonagy \& Target, 2000; Graña, 2007; Levy, 2005). Quando tais características contextuais estarão associadas à organização de personalidade borderline na adolescência? Essa possibilidade é apresentada nos estudos discutidos a seguir.

\section{Adolescência e organização de personalidade borderline}

Há muitas controvérsias no que se refere às definições de "condições psicopatológicas" no período da adolescência, já que é muito sutil a barreira que separa o "normal" e o "patológico" neste momento evolutivo. O adolescente está ainda em processo de formação de sua personalidade, bastante atravessado pelo contexto em que se encontra - portanto torna-se difícil falar em "estrutura" ou "transtorno de personalidade".

Enquanto alguns autores são categóricos em caracterizar transtornos de personalidade na infância e adolescência (Kernberg, 1990, 2003), outros, como Giovachinni (1993), Masterson (1993) e Outeiral (1993) definem as situações patológicas na adolescência como "estados" ou "organizações". Nesta mesma linha, Bergeret (1996/1998) postula as anestruturações.

A questão da possibilidade diagnóstica da personalidade borderline na adolescência (e suas manifestações) tem sido o foco de alguns estudos (Bleiberg, 1994; Bradley, Zittel, \& Westen, 2005; Chabrol, Chouicha, Montovany, \& Callahan, 2001; Chabrol e cols., 2004; Crick, Murray-Close, $\&$ Woods, 2005; Paris, 2005). Essas pesquisas sugerem que se deve considerar seriamente o diagnóstico de personalidade borderline na adolescência sempre que os pacientes apresentarem as características clássicas desta desordem. 
Os sintomas mais freqüentes encontrados nestes adolescentes, segundo a pesquisa de Chabrol e cols. (2001), foram ideação paranóide ou sintomas dissociativos $(97,1 \%)$, instabilidade afetiva $(88,6 \%)$, sentimento intenso de raiva $(85,6 \%)$, comportamentos autodestrutivos ou suicidas (82,9\%), esforços imensos para evitar o abandono (77\%), impulsividade $(65,7 \%)$, relacionamentos instáveis e intensos $(62,9 \%)$, distúrbio da identidade $(60 \%)$, e o sentimento de vazio $(57,1 \%)$. Dessa forma, apesar do continuum entre normalidade e patologia na adolescência, a personalidade borderline parece representar uma situação específica neste período (Bradley, Jenei, \& Westen, 2005; Crick e cols., 2005; Kernberg, 2003; Paris, 2005).

No que remete à concepção psicodinâmica da organização de personalidade borderline na infância e adolescência, os autores tendem a ter um consenso sobre este funcionamento psíquico e sobre suas vicissitudes. Assim, pensa-se em uma personalidade marcada por fragilidades nas vinculações afetivas, uso de defesas primitivas, dificuldades acentuadas no processo de individuação, difusão de identidade (falta de integração do conceito de self e de outros significativos) e nível de operações defensivas primitivas (centradas no mecanismo de clivagem e identificação projetiva). Soma-se a isso a falta constitucional de autonomia primária, a baixa tolerância à ansiedade, o excessivo desenvolvimento de impulsos agressivos e a vivência de uma realidade que produz excesso de frustração nestes adolescentes (Baird e cols., 2005; Bradley \& Westen, 2005; Cardoso, 2005; Graña, 2007; Kernberg, 1995; Kernberg, Selzer, Koenigsberg, Carr, \& Appelbaum, 1991; Levy, 2005; Maranga, 2002).

Destacam-se, ainda as dificuldades nos relacionamentos interpessoais (acentuadamente conturbados e instáveis), as angústias de cunho depressivo (depressão narcísica) e um funcionamento marcadamente impulsivo e atuador. A intolerância à frustração, a precariedade na capacidade sublimatória, a falta de constância objetal e as falhas narcísicas estão presentes nesta dinâmica, acarretando graves perturbações na construção identitária e nas relações de objeto (denotando uma indiscriminação self-objeto). A tênue integração do superego também é um fator relevante, resultando em tendências anti-sociais e predomínio do processo primário (Agrawal e cols., 2004; Bradley \& Westen, 2005; Bradley e cols., 2005; Cardoso, 2005; Carvalho, 2004; Diguer e cols., 2004; Figueiredo, 2003; Kernberg, 2003; Outeiral, 1993; Vaz \& Santos, 2006; Villa \& Cardoso, 2004; Westen, Ludolph, Lerner, Ruffins, \& Wiss, 1990; Zilberleib, 2006).

Além disso, estão presentes dificuldades em reconhecer, diferenciar e integrar emoções, assim como representações fragmentadas e malevolentes de si e dos outros, além de características comportamentais - suicídio e auto-agressão, uso de substâncias, bulimia. Tais situações trazem, como conseqüências, confusões de pensamentos e sentimentos, dificuldades com os limites, fronteiras frágeis, fluidas e dificuldades em manter relações íntimas e duradouras com as pessoas. Nesse sentido, a gestão das tormentas afetivas mostra-se fundamental nos adolescentes com esta organização de personalidade (Agrawal e cols., 2004; Bradley \& Westen, 2005; Kernberg, 2003; Westen, Muderrisoglu, Shedler, Fowler, \& Koren, 1997).

A questão do senso de identidade dos adolescentes borderline é explorada em vários estudos (Bradley \& Westen, 2005; Bradley, Zittel e cols., 2005; Fonagy \& Target, 2000; Kernberg, 1995). Os autores afirmam que a falta de uma identidade integrada é uma característica essencial na organização borderline de personalidade, repercutindo na experiência subjetiva de vazio crônico e percepções de si e dos outros contraditórias, empobrecidas e superficiais. $\mathrm{O}$ self é visto como inadequado, não-louvável e indigno pelos adolescentes borderline. Além disso, estes adolescentes referem se sentir como um estranho, inferiorizados, impotentes, enxergando-se como permanentemente danificados, fragilizados, "do mal". Disso decorre a sensibilidade à rejeição, o temor à solidão e o conflito entre uma desesperada necessidade de conexão com os outros (para regular seus afetos e seus medos) e os sentimentos de raiva e ódio, decorrentes de sensações de rejeição, incompreensão ou vitimização por parte destes adolescentes (Bradley e cols., 2005; Bradley \& Westen, 2005; Gunderson, 1996; Putnam \& Silk, 2005).

Kernberg (1995) levanta o questionamento relativo às dificuldades em diferenciar os graus normais e patológicos de difusão de identidade na adolescência. $\mathrm{O}$ autor conclui que a diferença está na "capacidade do adolescente não-borderline de experenciar a culpa e preocupação, de estabelecer relações interpessoais duradouras e não-exploradoras (...) e de avaliar realisticamente e em profundidade as pessoas" ( $\mathrm{p}$. 53). Assim, os sujeitos borderline, no final da adolescência, se diferenciam por não realizarem as tarefas desenvolvimentais típicas dessa etapa, especialmente a consolidação de um senso de identidade do ego, a afirmação da identidade sexual, o afrouxamento dos laços com as figuras parentais e a superação da regência pelo superego infantil.

Diante da complexidade dos aspectos representacionais e das vicissitudes da constituição psíquica dos adolescentes borderline, constata-se a importância da compreensão de suas experiências e história familiar, além das relações existentes entre as características das vinculações afetivas entre pais e filhos e os padrões de apego estabelecidos e a sua saúde mental e/ou psicopatologia. Os estudos identificados nessa categoria são apresentados a seguir.

\section{Os vínculos afetivos dos adolescentes com organização de personalidade borderline}

A história familiar e os vínculos afetivos ocupam um lugar de destaque na psicodinâmica dos adolescentes borderline. A literatura tem enfatizado os antecedentes desenvolvimentais dessa organização de personalidade, discutindo o papel das experiências traumáticas, como abuso sexual e físico, história de prolongadas separações precoces e perdas parentais (como divórcio, doenças, viagens), exposição a 
atitudes parentais dominadoras, frias afetivamente e sádicas, marcadas por negligência emocional, superproteção e controle excessivo. Nessa senda, aparece um contexto familiar instável e caótico, marcado por ansiedade de separação, negação da autonomia dos filhos e um modelo de comunicação baseado em atuações (em detrimento das palavras). Geralmente, trata-se de interações afetivas patológicas (diádicas, narcísicas, superprotetoras e/ou negligentes), relacionadas a padrões de apego inseguro (Agrawal e cols., 2004; Bradley \& Westen, 2005; Cardoso, 2005; Fruzzetti, Shenk, \& Hoffman, 2005; Graña, 2007; Hegenberg, 2000; Kernberg, 2003; Levy, 2005; Maranga, 2002; Outeiral, 1993; Zanarini e cols., 1997).

As pesquisas sobre o funcionamento dos adolescentes borderline e suas famílias têm privilegiado o uso de métodos quantitativos. Trata-se, predominantemente, de trabalhos internacionais. É freqüente o uso de questionários para a compreensão das características e dificuldades inerentes a esta organização de personalidade (Barone, 2003; Bernstein, Cohen, Skodol, Bezirganian, \& Brook, 2002; Chabrol e cols., 2004; Harvey \& Byrd, 2000; Helgeland \& Torgerser, 2004; Nickell, Waudby, \& Trull, 2002; Reich \& Zanarini, 2001; Rosenstein \& Horowitz, 1996; Russ e cols., 2003).

Por outro lado, algumas pesquisas qualitativas têm contribuído para o estudo dessa temática, possibilitando uma análise em profundidade de sua psicodinâmica. Aí se situam as pesquisas com materiais clínicos (artigos teóricoclínicos) e com utilização de técnicas projetivas (Romaro \& Loreiro, 1997; Silva \& Yazigi, 2004; Westen, Ludolph, Lerner, Ruffins, \& Wiss, 1990; Zilberleib, 2006).

Estudos de revisão de literatura também têm trazido acréscimos na elucidação das questões que permeiam a psicodinâmica dos adolescentes borderline e seus estilos de vinculações afetivas (Baird e cols., 2005; Bradley \& Westen, 2005; Fruzzetti, Shenk, \& Hoffman, 2005; Lenzenweger \& Cicchetti, 2005). Dentre as pesquisas teóricas sobre o tema, algumas têm focalizado as dimensões das relações de apego destes pacientes (Agrawal e cols., 2004; Barone, 2003; Bateman \& Fonagy, 2003; Holmes, 2003, 2004; Levy, 2005).

Na linha de estudos qualitativos, a partir da utilização do TAT (Teste de Apercepção Temática) e do Rorschach, alguns autores têm tratado das dimensões da identidade e das relações objetais dos pacientes borderline, sugerindo um funcionamento permeado por fragmentações, desvalorizações, manipulações, necessidade de gratificações dos objetos, impulsividade e dificuldades egóicas. Além disso, tendência a pensar de forma egocêntrica, apresentando dificuldades em diferenciar a sua perspectiva da dos outros (Romaro \& Loireiro, 1997; Silva \& Yazigi, 2004; Vaz \& Santos, 2006; Westen e cols., 1990; Zilberleib, 2006).

Alguns autores discutem as dificuldades na capacidade de mentalização (reconhecimento do estado mental do outro e do seu próprio estado mental) por parte dos pacientes borderline (Fonagy, 2000; Fonagy \& Target, 2000; Fonagy,
Target, Gergely, Allen, \& Bateman, 2003). Segundo eles, a diminuição da capacidade reflexiva destes pacientes dá-se em função dos padrões de apego inseguro em seus vínculos parentais, a partir de vivências de relações objetais primárias comprometidas (em especial na fragilidade da função especular que deveria ter sido exercida pelos cuidadores). Dessa forma, os "maus-tratos" sofridos por estes pacientes impedem a "mentalização" já que se torna perigoso reconhecer a intenção dos pais, pois isso faz com que se sintam sem valor, não dignos de amor. Assim, acabam tornando-se hipervigilantes em relação aos outros, tentando adaptar-se a eles, mas sem conseguir apreender seus próprios estados mentais.

No que se refere à associação da organização borderline com um contexto de relações familiares comprometidas, algumas pesquisas discutiram o papel das funções maternas e paternas nas manifestações deste sofrimento. A transmissão intergeracional de estilos parentais já foi salientada por Weber, Selig, Bernardi e Salvador (2006).

Um dos problemas centrais dos pacientes borderline seria a falha no desenvolvimento da constância objetal e a incapacidade de evocar imagens positivas e confortantes dos outros diante de situações ansiogênicas. Tais dificuldades seriam resultantes das inconsistências maternas (falhas ambientais) e falta de empatia em relação à criança. A psicopatologia borderline derivaria, então, da ineficácia na introjeção de um holding adequado e tranquilizador, pela falta de um ambiente cuidador e protetivo, resultando em uma intensa ansiedade de separação e ameaça de aniquilamento (Adler \& Buie, 1979; Beresin, 1994; Bleichmar, 1993/1994; Fonagy e cols., 2003; Reich \& Zanarini, 2001).

Levy (2005) realizou uma revisão de pesquisas acerca das implicações dos vínculos afetivos para a organização da personalidade borderline. O autor concluiu que há evidências substanciais sugerindo alguns fatores de risco para o desencadeamento desse quadro: vida familiar caótica, acrescida de alto nível de estresse parental e comunicações disruptivas entre os cuidadores e as crianças. Tais fatores interagem com o temperamento e predisposições genéticas como impulsividade, afeto negativo e propensão à agressão. A relação entre os fatores constitucionais e ambientais precisa ser considerada, portanto, no entendimento do funcionamento borderline.

Grande parte das pesquisas que tratam de relacionamentos pais-filhos em adolescentes borderline utilizam-se, enquanto embasamento, da teoria do apego, a partir dos pressupostos de Bowlby (1979/1997, 1976/1995), Ainsworth (1989) e Main (1988). Por exemplo, Nickell e cols. (2002) realizaram um estudo que mostrou que o vínculo afetivo e o estilo de apego - especialmente apego inseguro, ansioso ou ambivalente, e a percepção de uma relativa falta de cuidado e proteção da figura materna - estão associados com organizações borderline na infância e adolescência. A conclusão dessa pesquisa foi que os pacientes borderline apresentam dificuldades na esfera das relações íntimas e conflitivas no que se refere à dinâmica dependência versus distanciamento afetivo das pessoas. Mais especificamente, as disfunções nos padrões de 
apego refletem dificuldades em manejar a ansiedade, o que afeta as relações interpessoais e pode se manifestar como instabilidade afetiva, sentimentos extremos de raiva e ódio e comportamentos autodestrutivos.

Em concordância, para Atienza e Rodríguez (2004) o estilo de apego desorganizado tem sido o mais consistentemente associado com psicopatologia na infância e adolescência, estando relacionado com o desenvolvimento da organização borderline. Crianças e adolescentes com este estilo de apego apresentam condutas confusas e desordenadas, dificuldades de separação em relação à figura de apego, ausência de estratégias na busca de conforto diante de situações de estresse e atitudes ambivalentes e evitativas. Suas mães com freqüência foram vítimas de traumas, sendo ansiosas e temerosas. Projetam seus medos nas circunstâncias atuais e são incapazes de reconhecer as demandas de seu filho, oferecendo respostas inconsistentes. Os autores referem, ainda, que as conseqüências deste padrão de apego são pouco conhecidas, mas que se associa com ambientes familiares marcados por fatores de risco aumentados, como depressão materna, conflitos maritais ou maus-tratos e abuso.

Agrawal e cols. (2004) corroboram a afirmação de que relações de apego inseguro (principalmente do tipo desorganizado), durante a infância, mostram-se como um fator de risco significativo para a personalidade borderline, tendo em vista que todos os estudos por eles revisados associam o diagnóstico de personalidade borderline com estilos de apego inseguro. Porém, alertam para o fato da existência de diversos instrumentos que se prestam a medir e avaliar o apego, o que faz com que a subclassificação de apego inseguro varie entre as pesquisas (evitativo, preocupado, não-resolvido, desorganizado e temeroso são os mais citados).

Além disso, estudos têm avaliado o papel da negligência e da superproteção na infância dos pacientes borderline, além das representações parentais destes pacientes (Hallie \& Paris, 1991; Levy, 2005; Nickel e cols., 2002). Destacamse, nesse sentido, as percepções contraditórias, fragmentadas e conflitivas em relação aos pais. As mães geralmente são vistas como pouco carinhosas e pouco cuidadoras, altamente controladoras, com altos níveis de encorajamento de autonomia e alta permissividade, o que acaba refletindo em uma representação de falta de controle parental. Assim, famílias não abastecedoras, instáveis, imprevisíveis e com história de abuso intrafamiliar foram associadas ao desenvolvimento da organização borderline em adolescentes (Lyons-Ruth, Yellin, Melnick, \& Atwood, 2005; Reich \& Zanarini, 2001; Westen e cols., 1990; Zanarini e cols., 1997).

Aponta-se, ainda, que as falhas ocorridas durante a constituição psíquica destes adolescentes associam-se às suas interações precoces, podendo estar relacionadas às falhas na constituição psíquica da própria mãe. $\mathrm{O}$ fracasso da constelação narcisista da mãe impediria que ela pudesse exercer a função de objeto materno narcizizante, não conseguindo dar sentindo às experiências do bebê. Assim, as vivências não conseguiriam se transformar em experiências, e o sujeito se expressaria a partir de atuações e descargas, em detrimento das representações e simbolizações (Bleichmar, 1993/1994; Fonagy e cols., 2003; Graña, 2007; Outeiral, 1993). Daí decorrem as dificuldades nos vínculos afetivos dos adolescentes borderline: tornam-se invasivos, provocativos, sem limites claros entre o eu e o outro e buscam testar as pessoas com as quais se relacionam a fim de se certificarem dos vínculos (André, 2004; Villa \& Cardoso, 2004).

A organização borderline de personalidade também tem sido relacionada a experiências traumáticas vivenciadas na infância (Baird e cols., 2005; Fonagy e cols., 2003; Reich \& Zanarini, 2001). Ainda existem controvérsias no que diz respeito a esta associação: enquanto alguns estudos têm apontado uma relação bastante significativa entre experiências de trauma e o desenvolvimento de personalidade borderline, outros têm buscado romper com esta associação.

No primeiro grupo, já foi referido que a parentalidade inapropriada ou negligente e as experiências traumáticas estão associadas à etiologia do borderline. Abusos (físicos e sexuais), negligências, cuidados parentais empobrecidos, ambiente emocional primitivo imprevisível, psicopatologia parental, alcoolismo parental, assim como déficits nos fatores protetivos (talentos artísticos, desempenho escolar, habilidades) podem contribuir substancialmente para o desencadeamento da personalidade borderline (Barone, 2003; Beresin, 1994; Bradley, Jenei, \& Westen, 2005; Fruzzetti e cols., 2005; Helgeland \& Torgerser, 2004; Nickell e cols., 2002; Reich \& Zanarini, 2001; Russ e cols., 2003).

Por outro lado, discutindo a relação de causalidade entre as duas variáveis, abuso sexual e personalidade borderline, algumas pesquisas discutem o fato de que um significativo percentual dos pacientes avaliados como sendo borderline não tiveram situações de abuso físico e/ou sexual em sua infância. Deste modo, a evidência disponível não sustenta este fator (abuso) ou outros fatores ambientais como suficientes para explicar o desenvolvimento de personalidade borderline (Bradley, Jenei e cols., 2005; Fruzzetti e cols., 2005; Zanarini e cols., 1997).

Dessa forma, a presença de alguma forma de violência é um fator relevante na etiologia do borderline, mas tais ocorrências durante a infância parecem contribuir parcialmente na predição da personalidade borderline, dependendo da influência do ambiente familiar. A psicopatologia parental, bem como os cuidados parentais, parecem ter efeitos mais significativos no desenvolvimento dessa organização de personalidade.

\section{Considerações finais}

Algumas reflexões se destacam a partir da literatura revisada. Em primeiro lugar, as características da cultura e da sociedade contemporânea parecem constituir, muitas vezes, um terreno fértil para a potencialização de alguns aspectos que, como vimos, estão presentes na dinâmica da organização borderline. A ênfase no individualismo, no narcisismo, a 
indiferenciação entre as gerações e a fragilização dos vínculos afetivos são condições que podem facilitar a emergência de manifestações de sofrimento psíquico, incluindo aquelas situadas no espectro dos estados limítrofes.

Neste panorama, o sofrimento psíquico dos adolescentes tem se mostrado predominantemente associado com fenômenos de passagens ao ato. Dentre essas manifestações, os funcionamentos borderline vêm ganhando largo espaço, diante da instabilidade, vulnerabilidade, incertezas e inseguranças decorrentes do contexto social e familiar atual. $\mathrm{O}$ desamparo, a falta de ancoragem simbólica e as desestruturações cada vez mais intensas dos vínculos têm contribuído, em alguma medida, para tais sofrimentos.

Destacam-se, nestas organizações, as dificuldades na identidade e nas relações objetais. Além disso, uma psicodinâmica em que habitam situações de descontroles afetivos, sentimentos de raiva e ódio, intolerância à frustração e à solidão e fragilidades na capacidade reflexiva.

É importante destacar a dificuldade e a cautela que se faz necessária ao se considerar a hipótese de uma organização de personalidade borderline na adolescência. $\mathrm{O}$ fato de tratar-se de um período de transição, marcado por flutuações e imprecisões, e por um reordenamento pulsional e reorganização da identidade, comportamento impulsivo, entre outros aspectos, justifica essa cautela e os estudos revisados deixam isso evidente.

Embora a literatura existente sobre as organizações borderline na infância e na adolecência venha ganhando destaque no contexto científico, ainda muito do que se sabe sobre esse modo do funcionamento é fruto de investigações com pacientes adultos. Nesse sentido, urge a necessidade de mais pesquisas acerca desse tema, com estas populações, a fim de proporcionar maiores esclarecimentos sobre suas características e vicissitudes.

Soma-se a isso a constatação de que a maioria dos estudos que se debruçaram sobre esse tema é oriunda de fontes internacionais, carecendo-se, dessa forma, de pesquisas com as crianças e adolescentes brasileiros com organização borderline de personalidade. Além disso, os métodos quantitativos predominam na literatura referente a essa temática, buscando elucidar os principais fatores desencadeantes desse funcionamento e os instrumentos que se prestam a medir as características do mesmo.

Sem deixar de considerar as contribuições que tais estudos têm trazido, ressalta-se a importância de explorações qualitativas, prezando pelo estudo aprofundado de casos e compreensão das singularidades dessa organização de personalidade na infância e adolescência. Assim, estudos clínicos poderiam oferecer uma contribuição fundamental.

Deve-se salientar algumas limitações da presente revisão de literatura. A opção por determinadas bases de dados, e por um referencial teórico em particular, pode ter deixado de lado estudos que abordaram essa mesma temática a partir de outros vértices, que devem contribuir também para sua elucidação. O fato de se tratar de uma revisão não sistemática contribui para essa limitação.

Entretanto, a importância dos vínculos afetivos para a organização de personalidade borderline foi identificada na literatura revisada, respondendo ao objetivo do presente estudo. As pesquisas têm explorado especialmente as características dos vínculos de apego desses indivíduos, identificando uma associação entre organização de personalidade borderline e apego inseguro, especialmente do tipo inseguro desorganizado.

Há resultados que apontam também para a importância da presença de psicopatologia materna na história familiar desses casos, e algumas controvérsias sobre o papel que ocupam as experiências traumáticas eventualmente presentes. Esses aspectos reforçam a importância dos vínculos afetivos, na medida em que contribuem sobremaneira para a sua configuração.

A partir da revisão realizada, percebe-se que a questão dos vínculos afetivos especificamente dos adolescentes ainda é pouco discutida, carecendo de mais pesquisas. O que se tem sugerido, nesse ponto, é que a história familiar dos adolescentes com organização borderline é freqüentemente marcada por situações de desamparo, fragilidades no suporte familiar, vivências de abusos das mais diversas ordens e dinâmicas familiares bastante instáveis, desorganizadas, em que predominam estilos de apego inseguro. A falta de coerência e de estabilidade na identidade e nas relações objetais e as fragilidades nos vínculos afetivos desses jovens parecem ser suas características mais marcantes.

Embora alguns estudos tenham se dedicado à relação entre a organização borderline e os padrões de apego na infância e adolescência, permanece a necessidade de se compreender em que medida e de que forma as histórias de vida e as características dos vínculos afetivos destes adolescentes contribuem para as manifestações desse sofrimento. Ou seja, examinar como e em que medida as relações objetais destes adolescentes se interligam com o desenvolvimento de uma organização borderline de personalidade. Estudos longitudinais poderiam beneficiar esta discussão.

\section{Referências}

Adler, G., \& Buie, D. H. (1979). Aloneness and borderline psychopathology: The possible relevance of child developmental issues. International Journal of Psychoanalisis, 60, 83-96.

Agrawal, H. R., Gunderson, J., Holmes, B. M., \& Lyons Ruth, K. (2004). Attachment studies with Borderline patients: A review. Harvard Review of Psychiatry, 12, 94-104.

Ainsworth, M. D. (1989). Attachments beyond infancy. American Psychologist, 44, 709-716.

American Psychiatric Association. (1995). DSM-IV: Manual Diagnóstico e Estatístico de Transtornos Mentais (D. Batista, Trad., 4a ed.). Porto Alegre: Artes Médicas. 
André, J. (2004). Transferência borderline. In M. R. Cardoso (Org.), Limites (pp. 71-80). São Paulo: Escuta.

Atienza, D., \& Rodríguez, B. (2004). El vínculo y psicopatología en la infancia: Evaluación y tratamiento. Revista de Psiquiatría y Psicología del Niño y del Adolescente, 4(1), 82-90.

Baird, A. A., Veague, H. B., \& Rabbitt, C. E. (2005). Developmental precipitants of borderline personality disorder. Development and Psychopathology, 17, 10311049.

Barone, L. (2003). Developmental protective and risk factors in borderline personality disorder: A study using the Adult Attachment Interview. Attachment \& Human Development, 5, 64-77.

Bateman, A. W., \& Fonagy, P. F. (2003). The development of attachment-based treatment program for borderline personality disorder. Bulletin of Menninger Clinic, 67, 187-211.

Berenstein, I. (2003). Reflexões sobre uma psicanálise do vínculo. In A. Green (Org.), Psicanálise contemporânea: Revista Francesa de Psicanálise, Número especial, 2001 (pp. 183-198). Rio de Janeiro: Imago.

Beresin, E. (1994). Developmental formulation and psychotherapy of borderline adolescents. American Journal of psychotherapy, 48, 5-28.

Bergeret, J. (1998). Personalidade normal e patológica (M. E. V. Flores, Trad.). Porto Alegre: Artes Médicas. (Original publicado em 1996)

Bernstein, D. P., Cohen, P., Skodol, A. M. D., Bezirganian, S. M. D., \& Brook, J. S. (2002). Childhood antecedents of Adolescent Personality Disorders. Journal of Consulting and Clinical Psychology, 70, 6-20.

Birman, J. (2005). Mal-estar na atualidade: Psicanálise e as novas formas de subjetivação (5a ed.). Rio de Janeiro: Civilização Brasileira.

Bleiberg, E. (1994). Borderline disorders in children and adolescents: The concept, the diagnosis and the controversies. Bulletin of the Menninger Clinic, 58, 169196.

Bleichmar, S. (1994). A fundação do inconsciente: Destinos de pulsão, destinos do sujeito (K. B. Behr, Trad.). Porto Alegre: Artes Médicas. (Original publicado em 1993)

Bowlby, J. (1997). Formação e rompimento de laços afetivos (A. Cabral, Trad.) São Paulo: Martins Fontes. (Original publicado em 1979)

Bowlby, J. (1995). Cuidados maternos e saúde mental (V. L. B. Souza \& I. Rizzini, Trad.) São Paulo: Martins Fontes. (Original publicado em 1976)

Bradley, R., \& Westen, D. (2005). The psychodynamics of borderline personality disorder: A view from developmental psychophathology. Development and Psychopathology, 17, 927-957.

Bradley, R., Jenei, J., \& Westen, D. (2005). Etiology of borderline personality disorder: Disentangling the contributions of intercorrelated antecedents. Journal of Nervous and Mental Disease, 193, 24-31.
Bradley, R., Zittel, C., \& Westen, D. (2005). The borderline personality diagnosis in adolescents: Gender differences and subtypes. Journal of Child Psychology and Psychiatry, 46, 1006-1019.

Calligaris, C. (2000). A adolescência: São Paulo: Publifolha.

Cardoso, M. R. (2005). A servidão ao "outro" nos estados limites. Psychê, 9, 65-75.

Carvalho, M. T. M. (2004). Sobre o alcance e os limites do recalcamento nas chamadas "psicopatologias da contemporaneidade". In M. R. Cardoso (Org.), Limites (pp. 151-167). São Paulo: Escuta.

Chabrol, H., Chouicha, K., Montovany, A., \& Callahan, S. (2001). Symptomatology of DSM IV borderline personality disorder in a non-clinical sample of adolescents: Study of 35 borderline cases. L'Encephale, 27, 120-127.

Chabrol, H., Montovany, A., Ducongé, E., Kallmeyer, A., Mullet, E. \& Leichsenring, F. (2004). Factor structure of the borderline personality inventory in adolescents. European Journal of Psychological Assessment, 20, 5965.

Crick, N., Murray-Close, D., \& Woods, K. (2005). Borderline personality features in childhood: A short-term longitudinal study. Development and Psychopathology, 17, 1051-1070.

Diguer, L., Pelettier, S., Hébert, E., Descôteaux, J., Rousseau, J. P., \& Daoust, J. P. (2004). Personality organizations, psychiatric severity, and self and object representations. Psychoanalytic Psychology, 21, 259-275.

Figueiredo, L. C. (2003). Elementos para a clínica contemporânea. São Paulo: Escuta.

Fonagy, P. (2000). Apegos patológicos y acción terapéutica [Versão eletrônica]. Aperturas Psicoanalíticas, 4. Recuperado em 31 agosto 2003, de http://www.aperturas. org/4fonagy.html

Fonagy, P., \& Target, M. (2000). Playing with reality: III the persistence of dual psychic reality in borderline patients. International Journal of Psychoanalysis, 81, 853-876.

Fonagy, P., \& Target, M. (2004). Questões desenvolvimentais na adolescência normal e colapso na adolescência. In $\mathrm{R}$. Granã \& A. Piva (Org.), A atualidade da psicanálise de adolescentes: Formas do mal-estar na juventude contemporânea. São Paulo: Casa do Psicólogo.

Fonagy, P., Target, M., Gergely, G., Allen, J. G., \& Bateman, A. (2003). The developmental roots of borderline personality disorder in early attachment relationships. Psychoanalytic Inquiry, 23, 412-459.

Fruzzetti, A., Shenk, C., \& Hoffman, P. (2005). Family interaction and the development of borderline personality disorder: A transactional model. Development and Psychopathology, 17, 1007-1030.

Giovacchinni, P. (1993). Os aspectos borderline da adolescência e o estado borderline. In J. Outeiral (Org.), $O$ adolescente borderline (pp. 86-104). Porto Alegre: Artes Médicas. 
Graña, R. (2007). La psicopatología de la adolescencia y el espectro borderline [Versão eletrônica]. Revista de Psicoanálisis Aperturas Psicoanaliticas, 26. Recuperado em 22 março 2008, de http://www.aperturas.org/26grana. html

Green, A. (1977). The borderline concept. In G. Adler (Orgs.), Borderline personality disorders (pp. 15-44). New York: Peter Hartcollis.

Gunderson, J. G. (1996). The borderline patient's intolerance of aloneness: Insecure attachments and therapist availability. American Journal of Psychiatry, 153, 752758.

Gunderson, J. (2008). Borderline personality disorder: An overview. Social Work in Mental Health, 6, 5-12.

Hallie, Z., \& Paris, J. (1991). Parents' emotional neglect and overprotection according to the recollections of patients with borderline personality disorder. American Journal of Psychiatry, 148, 648-651.

Harvey, M., \& Byrd, M. (2000). Relationships between adolescents' attachment styles and family functioning. Adolescence, 35, 345-356.

Hegenberg, M. (2000). Borderline (Coleção Clínica Psicanalítica, Vol. 4). São Paulo: Casa do Psicólogo.

Helgeland, M., \& Togerser, S. (2004). Developmental atecedents of Borderline Personality Disorder. Comprehensive Psychiatry, 45, 138-147.

Holmes, J. (2003). Borderline personality disorder and the search for meaning: An attachment perspective. Australian and New Zealand Journal of Psychiatry, 37, 524-531.

Holmes, J. (2004). Disorganized attachment and Borderline Personality Disorder: A clinical perspective. Attachment \& Human Development, 6, 181-190.

Jeammet, P., \& Corcos, M. (2005). Novas problemáticas da adolescência: Evolução e manejo da dependência. São Paulo: Casa do Psicólogo.

Justo, J. S. (2005). O "ficar" na adolescência e paradigmas de relacionamento amoroso da contemporaneidade. Revista do Departamento de Psicologia - UFF, 17, 61-77,

Kaës, R., Faimberg, H., Enriquez, M., \& Barane, J. J. (2001). Transmissão da vida psíquica entre gerações (C. Berliner, Trad.). São Paulo: Casa do Psicólogo. (Original publicado em 1993)

Kancyper, L. (1999). Confrontação de gerações: Estudo psicanalítico (A. V. Fuzato, Trad.). São Paulo: Casa do Psicólogo.

Kehl, M. R. (2003). Em defesa da família tentacular. In G. C. Groeninga \& R. C. Pereira (Org.), Direito de família e psicanálise (pp. 163-178). Rio de Janeiro: Imago.

Kernberg, O., Selzer, M. A., Koenigsberg, H. W., Carr, A. C., \& Appelbaum, A. H. (1991). Psicoterapia psicodinâmica de pacientes borderline. Porto Alegre: Artes Médicas.

Kernberg, O. (1995) Transtornos graves de personalidade: Estratégias psicoterapêuticas. Porto Alegre: Artes Médicas.
Kernberg, P. (1990). Resolved: Borderline personality exists in children under twelve. Journal of Amerian Academy of Child and Adolescent Psychiatry, 29, 478-483.

Kernberg, P. (2003). Transtornos da personalidade em crianças e adolescentes. Porto Alegre: Artmed.

Leivi, M. (1995). Historización, actualidad y acción en la adolescência. Revista de la Associacion Psicoanalitica de Buenos Aires, 17, 585-611.

Lenzenweger, M., \& Cicchetti, D. (2005). Toward a developmental psychopathology approach to borderline personality disorder. Development and Psychopathology, 17, 893-898.

Levy, K. (2005). The implications of attachment theory and research for understanding borderline personality disorder. Development and Psychopathology, 17, 959986.

Levisky, D. L. (2002). Depressões narcísicas na adolescência e o impacto da cultura. Psychê, 6, 125-136.

Lyons-Ruth, K., Yellin, C., Melnick, S., \&Atwood, G. (2005). Expanding the concept of unresolved mental states: Hostile/helpless states of mind on the Adult Attachment Interview are associated with disrupted mother-infant communication and infant disorganization. Development and Psychopathology, 17, 1-23.

Main, M. (1988). Categories of response to reunion with the parents at age 6: Predictable from infant attachment classifications and stable over a month period. Developmental Psychology, 24, 415-426.

Maranga, R. (2002). Organizações borderline: Aspectos psicodinâmicos. Análise Psicológica, 20, 219-223.

Masterson, J. (1993) O adolescente borderline: Uma visão das relações de objeto. In J. Outeiral (Org.), O adolescente borderline (pp. 110-125). Porto Alegre: Artes Médicas.

Nickell, A. D., Waudby, C. J., \& Trull,T. J. (2002). Attachment, parental bonding and borderline personality disorder features in young adults. Journal of Personality Disorders, 16, 148-159.

Outeiral, J. (1993). O adolescente borderline. Porto Alegre: Artes Médicas.

Paris, J. (2005). Diagnosing borderline personality disorder in adolescence. Adolescent Psychiatry, 29, 237-247.

Putnam, K. M., \& Silk, K. R. (2005). Emotion dysregulation and the development of borderline personality disorder. Development and Psychopathology, 17, 899-925

Reich D. B., \& Zanarini, M. C. (2001). Developmental aspects of Borderline Personality Disorder. Harvard Review of Psychiatry 9, 295-301.

Romaro, R. A., \& Loreiro, S. R. (1997). Distúrbio de personalidade Borderline: Comparação em dois momentos da avaliação através da técnica de Rorschach. Boletim de Psicologia, 47, 73-89.

Rosenstein, D., \& Horowitz, H. (1996). Adolescent attachment and psychopathology. Journal of Consulting and Clinical Psychology, 64, 244-253. 
Roudinesco, E. (2003). A família em desordem (A. Telles, Trad.). Rio de Janeiro: Jorge Zahar.

Russ, E., Heim, A., \& Westen, D. (2003). Parental bonding and personality pathology assessed by clinician report. Journal of Personality Disorders, 17, 522-536.

Savietto, B. B. (2007). Passagem ao ato e adolescência contemporânea: Pais "desmapeados", filhos desamparados [Versão eletrônica]. Revista Latinoamericana de Psicopatologia Fundamental, 10, 438-453. Recuperado em 10 abril 2008, de http://www. fundamentalpsychopathology.org/anais2006/fedida/3. men.htm

Savietto, B. B., \& Cardoso, M. R. (2006). Adolescência: Ato e atualidade. Revista Mal-estar e Subjetividade, 6, 15-43.

Silva, J. F., \& Yazigi, L. (2004). Dois vértices da investigação de pacientes borderline: A clínica psicanalítica e a avaliação psicológica: Alice quebra-vidros. Revista Brasileira de Psicanálise, 38, 621-636.

Skodol, A. E., Gunderson, J. G., Pfohl, B., Widiger, T. A., Livesley, W. J., \& Siever, L. J. (2002). The borderline diagnosis I: Psychopathology, comorbidity, and personality structure. Biological Psychiatry, 51, 936950.

Vaz, C., \& Santos, M. A. (2006). Representação de objeto e organização psíquica: Integração dinâmica dos dados do Rorschach. Psico, 37, 249-261.

Villa, F. C., \& Cardoso, M. R. (2004). A questão das fronteiras nos estados limites. In M. R. Cardoso (Org.), Limites (pp. 59-70). São Paulo: Escuta.

Weber, L. N. D., Selig, G. A., Bernardi, M. G., \& Salvador, A. P. V. (2006). Continuidade dos estilos parentais através das gerações: Transmissão intergeracional de estilos parentais. Paidéia (Ribeirão Preto), 16, 407-414.

Westen, D., Ludolph, P., Lerner, H., Ruffins, S., \& Wiss, C. (1990). Object relations in borderline adolescents. Journal of American Academy of Child and Adolescent Psychiatry, 29, 338-348.

Westen, D., Muderrisoglu, S., Shedler, J., Fowler, C., \& Koren, D. (1997). Affect regulation and affective experience: Individual differences, group differences, and measurement using a Q-sort procedure. Journal of Consulting and Clinical Psychology, 65, 429-439.

Zanarini, M. C., Williams, A. A., Lewis, R. E., Reich, R. B., Vera, S. C., Marino, M. F., Levin, A., Yong, L., \& Frankenburg, F. R. (1997). Reported pathological childhood experiences associated with the development of borderline personality disorder. American Journal of Psychiatry, 154, 1101-1106.

Zilberleib, C. M. O. V. (2006). O acompanhamento terapêutico e as relações de objeto em pacientes-limites. Psychê, 10, 53-66.
Aline Bedin Jordão é psicóloga clínica e coordenadora técnica da Clínica de Estudos e Intervenções em Psicologia (CEIP) da Universidade Federal de Santa Maria (UFSM).

Vera Regina Röhnelt Ramires é Professora Titular do Programa de Pós-graduação em Psicologia Clínica da Universidade do Vale do Rio dos Sinos, UNISINOS. 